\title{
Near-field Occupational Exposure in FM Transmission Pylons
}

\author{
B. Fetouri( ${ }^{(1)}$, A. Ouberehil ${ }^{(2)}$, P. De Doncker ${ }^{(3)}$, and J. Wiart ${ }^{(1)}$ \\ (1) C2M Chair, Telecom Paris, Institut Ploytechnique de Paris, Palaiseau, France \\ (2) TDF, France \\ (3) Université Libre de Bruxelles, Belgium
}

\begin{abstract}
This paper provides a new method to generalize electromagnetic field exposure via surrogate modeling. The study focuses on linking maximum local specific absorption rate and field intensity in FM transmission towers.
\end{abstract}

\section{Introduction}

FM transmitters used in broadcasting are located on towers that workers have to climb in order to do maintenance and repair work. Occupational exposure to electromagnetic fields (EMF) must be monitored in these situations. ICNIRP recommended limits are made of basic restrictions (BRs) and reference levels. The fundamental limits are the BRs, established to limit the specific absorption rate (SAR) (1) that represents the absorbed power per unit mass of tissue in the human body. The BRs define the whole-body SAR (WBSAR) limits as well as the local SAR $\left(S A R_{10 g}\right)$ limits that quantify the SAR averaged over 10 grams of tissue. BRs assessment is impossible in-situ since they are too complex to measure. ICNIRP has therefore defined derived limits, the reference levels (RLs) in terms of electric field strength. They are conservative but often used because of their convenience. ICNIRP RLs are used as action values in Directive 2013/35/EU (2). Compliance to RLs guarantees compliance to WBSAR limits, but does not necessarily mean that maximum $S A R_{10 \mathrm{~g}}$ is compliant. Various papers have studied workers' occupational exposure on broadcasting towers (3) (4) (5) (6). In this paper, we will analyze the worker's exposure to near-field EMF from FM transmitters. The objective of this study is to find a relationship between WBSAR, $S A R_{10 g}$ and in-situ measurable field quantities, namely averaged and maximum field strength. The study will require the generalization of EMF exposure cases in perturbed nearfield environments, which has not been proposed before.

\section{Method}

Taking into account progress of EM solver, transmission pylons can be modeled with high details and electromagnetic fields (EMF) occurring in pylons can be computed and analyzed afterwards to assess electromagnetic exposure. Nevertheless a new case of exposure would require a new calculation and despite progress in computational codes, these calculations can request hours. Therefore models of all existing and possible pylons cannot be used by EMF solvers to establish the relationship between EMF and SAR. To overcome such limits, we proposed a computationally efficient way to generalize electromagnetic field exposure cases in transmission pylons.

The method we propose is to generate fields that are realistic, keeping those that can be found in actual transmission sites. Criteria have to be defined in order to judge the admissibility of produced EMF. We considered four criteria to assess electromagnetic fields inside pylons: angles between $\mathrm{E}$ and $\mathrm{H}$ fields, impedance $(|E| /|H|)$, intensity ratio $\left(|E|_{\text {averaged }} /|E|_{\max }\right)$ and spatial concentration around maximum field level.

The angles between $\mathrm{E}$ and $\mathrm{H}$ fields are usually equal to $\pi / 2$ for any far-field electromagnetic field, but occupational exposure is assessed in the near-field region of the antennas on the FM transmission sites. The wave impedance is also expected to be different from far-field. The field strength ratio will provide information on the field intensity distribution. The spatial concentration around maximum field level is the average distances between maximum field intensity and all values higher than the 90th percentile of fields' strength. A tunable near-field generator, based on fields emitted by set infinitesimal dipoles, was programmed to produce $\mathrm{E}$ and $\mathrm{H}$ fields based on the four above criteria.

Each infinitesimal dipole offers 7 degrees of freedom, 4 are linked to dipole position (coordinates $\mathrm{x}, \mathrm{y}$ and $\mathrm{z}$ ) and amplitude, and 3 to phase shift and dipole orientation. When assembled together, infinitesimal dipoles offer a near-field EMF distribution. The total number of degrees of freedom $\mathrm{d}$ depends on the number of dipoles: $d=$ $n_{\text {dipoles }} \times n_{\text {inputs }}=n_{\text {dipoles }} \times 7$.

The obtained fields must be characterized using the criteria mentioned earlier.

Since we are interested in human exposure, fields produced by the dipoles will be considered in "human volumes" (HVol) sized to humans, typically $0.7 \mathrm{~m} \times 0.4 \mathrm{~m}$ $\times 1.8 \mathrm{~m}$. A HVol will slide inside a pylon and a human vector (HVect) consisting of field analytics of the 4 criteria mentioned earlier is calculated for each new 
position. Human vectors will be used to plot CDF and PDF for every criterion.

$$
\text { HVect }=\left[\frac{|E|}{|H|} ; \frac{|E|_{\text {avg }}}{|E|_{\text {max }}} ; \text { angles } ; \text { concentration }\right]
$$

Components of the Hvect are measured every 10 centimeters inside the Hvol then averaged. $|E|_{\text {avg }}$ is the averaged field strength in $\mathrm{V} / \mathrm{m}$, averaged on the entire Hvol. $|E|_{\max }$ is the maximum field strength in $\mathrm{V} / \mathrm{m}$, measured on $10 \mathrm{~cm}$ sphere, with a $1 \mathrm{~cm}$ step.

One Hvect is calculated per Hvol. EMF in HVol are obtained using the tunable near-field generator. CDF and PDF of HVect are compared with distributions from pylons, using statistical methods of measuring differences between distributions shapes (Jensen-Shannon divergence and Kolmogorov-Smirnov test).

The equivalent principle is then used to compute SAR induced by EMF in Hvol via the Duke human model (7) and the well-known Finite difference in Time domain method (FDTD). Such an equivalent principle is implemented via a "Huygens box".

\section{Results \& Discussion}

Millions of cases of HVects have been generated using Monte Carlo simulations with random inputs. Distributions obtained with the generator have been compared to those obtained in pylons. Visualization of the distributions shows same behavior for the near-field generator for the four criteria when compared to simulated pylons, thus validating our near-field generator. 500 cases are randomly chosen from the selected distributions after the Hvect analysis. $500 \mathrm{WBSAR}$ and $S A R_{10 \mathrm{~g}}$ simulations were performed.

\subsection{Maximum $S A R_{10 g}$ results}

We proceed to calculate the maximum $S A R_{10 g}$ using the method described earlier. To analyze the relationship between the maximum $S A R_{10 g}$ and the couple $\left[|E|_{\text {max }} ;|E|_{\text {avg }}\right]$, we define

$$
k=\frac{S A R_{10 g}}{S A R_{10 \text { glimit }}}
$$

Where $S A R_{10 g}$ is the measured SAR obtained after a simulation and $S A R_{10 \text { glimit }}$ is defined in the basic restrictions, which are $10 \mathrm{~W} / \mathrm{kg}$ for head \& trunk or $20 \mathrm{~W} / \mathrm{kg}$ for limbs, depending on the position. As figure 1 shows, there is no clear relationship between maximum $S A R_{10 g}$ and $|E|_{\max }$.
Figure 2 shows that higher $|E|_{\text {avg }}$ values induce higher maximum local SAR values. This is because $|E|_{\text {avg }}$ affects global exposure and sets an exposure baseline that can only be increased by $|E|_{\max } .|E|_{\max }$ does not suffice on its own to predict maximum $S A R_{10 \mathrm{~g}}$.

The distribution of the $\mathrm{k}$ ratio for each case can be seen in figure 2 for the calculations we performed. $S A R_{10 g}$ distribution for maxima located both on head \& trunk or limbs is represented on said figure.

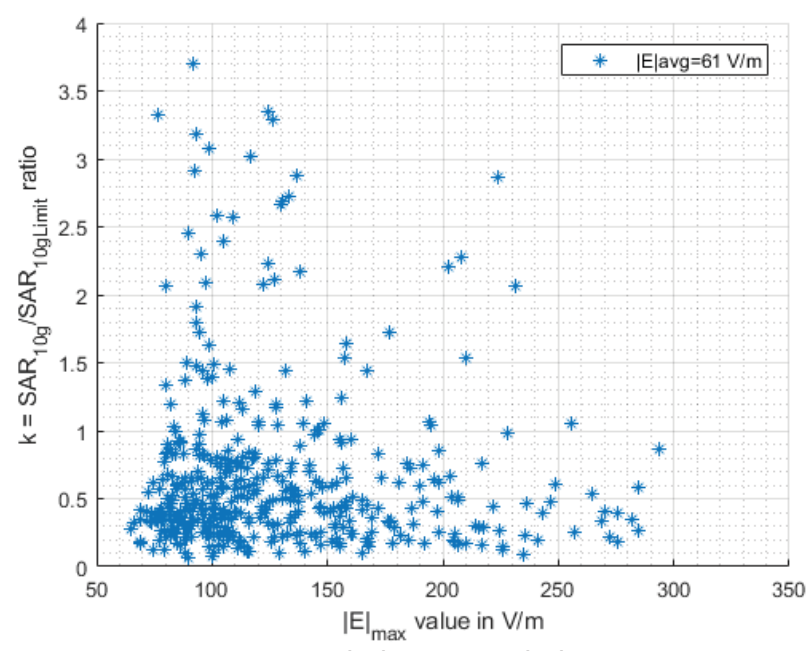

Figure 1. $k$ ratio versus $|E|_{\max }$ with $|E|_{\text {avg }}=61 \mathrm{~V} / \mathrm{m}$.

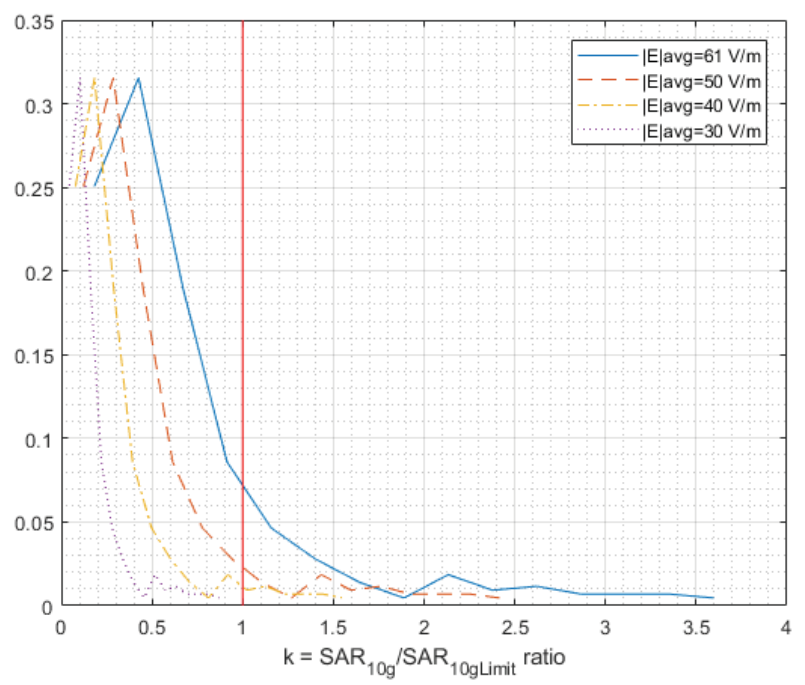

Figure 2. $S A R_{10 g}$ ratio PDF for different $|E|_{\text {avg }}$ values.

Table 1. Compliance rate for different $|E|_{\text {avg }}$ values.

\begin{tabular}{cc}
\hline$|\boldsymbol{E}|_{\text {avg }}(\mathrm{V} / \mathrm{m})$ & Compliance rate \\
\hline 61 & $84 \%$ \\
50 & $91 \%$ \\
40 & $96 \%$ \\
30 & $100 \%$ \\
\hline
\end{tabular}

According to the 500 cases' results in figure 2, we can see that while some $S A R_{10 g}$ simulations have a $\mathrm{k}$ ratio over the $k=1$ threshold, $84 \%$ of all cases were under the threshold when $|E|_{\text {avg }}=61 \mathrm{~V} / \mathrm{m}$. Table 1 shows 
$|E|_{\text {avg }}=30 \mathrm{~V} / \mathrm{m}$ guarantees a $100 \%$ compliance rate for all 500 cases we simulated.

\subsection{WBSAR results}

WBSAR results will depend mostly on $|E|_{\text {avg }}$, as this quantity is an indicator of global exposure in a Hvol. Figure 3 shows WBSAR is a function of $|E|_{\text {avg }}$ with some variation. The figure suggests a high correlation between WBSAR and $|E|_{\text {avg }}$.

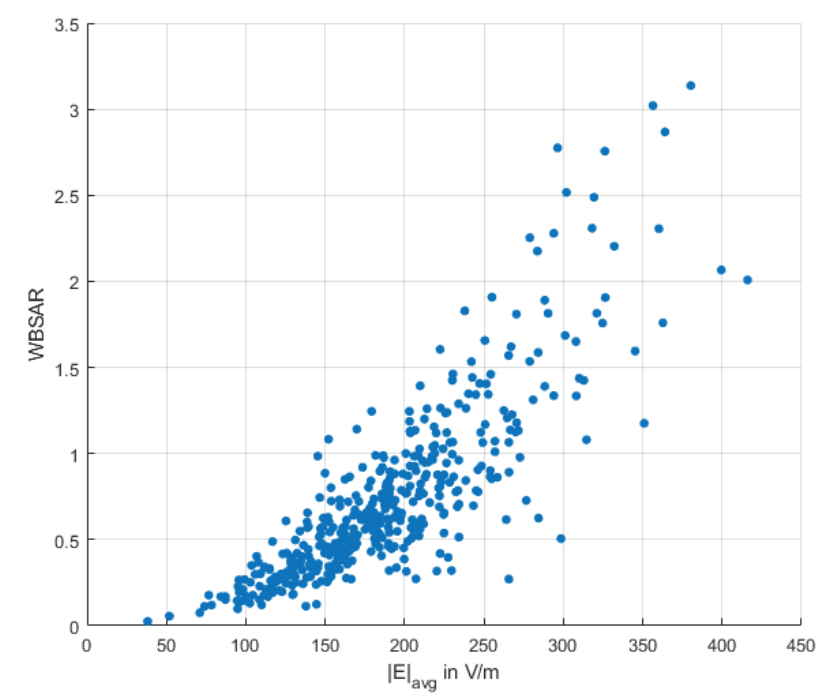

Figure 3. WBSAR versus $|E|_{\text {avg }}$.

$|E|_{\max }$ 's impact should be minimal, as it is a positionrelated quantity averaged over a $10 \mathrm{~cm}$ cube. Figure 4 shows higher $|E|_{\max }$ values do not necessarily yield a higher WBSAR, confirming our hypothesis.

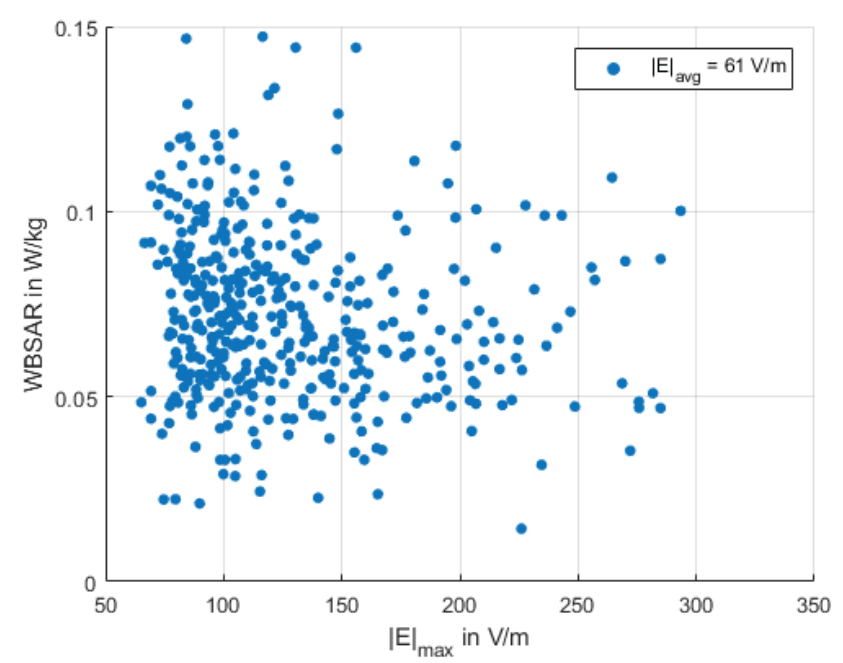

Figure 4. WBSAR versus $|E|_{\max }$ for $|E|_{\text {avg }}=61 \mathrm{~V} / \mathrm{m}$.

\section{Conclusion}

This paper proposed a new method to characterize and analyzed electromagnetic fields inside pylons in their near-field complexity with the help of the human vector decomposition. We have created a near-field generator to reproduce fields that can be found in pylons. FDTD calculations have were performed to compute local \& whole-body specific absorption rate (SAR) in 500 random cases of exposure. The electric field's impact on local \& whole-body SAR was studied. The study has provided upper bounds for local SAR results and showed a $100 \%$ compliance rate when the averaged electric field strength is $30 \mathrm{~V} / \mathrm{m}$ or less, thus providing a quantity that can be measured in-situ for local SAR assessment. Future works will look at surrogate modeling to provide estimation formulas for both local \& whole-body SAR.

\section{References}

1. Guidelines for limiting exposure to time-varying electric, magnetic and electromagnetic fields. ICNIRP. 1998, Health Phys.

2. Directive 2013/35/EU of The European Parliament and of The Council on the minimum health and safety requirements regarding the exposure of workers to the risks arising from physical agents. EUR-Lex. [En ligne] 2013. [Citation : 7 March 2019.] https://eur-

lex.europa.eu/legalcontent/EN/TXT/HTML/?uri=CELEX:32013L0035\&fro $\mathrm{m}=\mathrm{EN}$.

3. Occupational exposure assessment on an FM mast: electric field and SAR values. B.Valic, Kos, B. and Gajsek, P. s.1. : Taylor \& Francis, 2012.

4. Bolte, Pruppers. Electromagnetic fields in the working environment. Ministry of Health, Welfare and Sport. [En ligne] [Citation : 0403 2019.]

https://www.rivm.nl/bibliotheek/rapporten/610015001.pdf

5. Jokela, Puranen. Occupational RF exposures. Radiat Prot Dosimetry. 1999, Vol. 83, 119-24.

6. Simultaneous Occupational Exposure to FM and UHF Transmitters. Valič, B., Kos, B. et Gajšek, P. 2, s.l. : Taylor \& Francis, 2012, International Journal of Occupational Safety and Ergonomics, Vol. 18.

7. The Virtual Family-development of surface-based anatomical models of two adults and two children. Christ, A., Kainz, W., Hahn, E. G., Honegger, K., Zefferer, M., Neufeld, E., Rascher, W., Janka, R., Bautz, W., Chen, J., Kiefer, B., Schmitt, P., Hollenbach, H., Shen, J., Oberle, M., Szczerba, D., Kam, A., Guag, J., Kuster, N. s.1. : IOP Publishing, 2010, Physics in Medicine and Biology. 